\title{
Evidence of Simultaneous Spectral Hole Burning Involving Two Tiers of the Protein Energy Landscape in Cytochrome bof.
}

Golia Shafiei ${ }^{1}$, Alexander Levenberg ${ }^{1}$, Maria A. Lujan ${ }^{2}$, Rafael Picorel ${ }^{2}$ and Valter Zazubovich ${ }^{1, *}$ ${ }^{1}$ Department of Physics, Concordia University, 7141 Sherbrooke Str. West, Montreal, Quebec H4B 1 R6 Canada; ${ }^{2}$ Estacion Experimental de Aula Dei (CSIC), Avda. Montañana 1005, 50059 Zaragoza, Spain.

\begin{abstract}
Cytochrome $b_{6} f$, with one chlorophyll molecule per protein monomer, is a simple model system whose studies help achieve better understanding of non-photochemical spectral hole burning (NPHB) and single-complex spectroscopy results obtained in more complicated photosynthetic chlorophyll-protein complexes. We are reporting new data and proposing an alternative explanation for spectral dynamics that was recently observed in Cytochrome $b_{6} f$ using NPHB [Najafi et al., J. Phys. Chem. B 2015, 119, 6930-6940]. The relevant distribution of the tunneling parameter $\lambda$ is a superposition of two components that are nearly degenerate in terms of the resultant NPHB yield and represent two tiers of the energy landscape responsible for NPHB. These two components likely burn competitively. However, similar values of the NPHB yield result from distinctly different combinations of barrier heights, shifts along the generalized coordinate $d$ and/or masses of the entities involved in conformational changes $m$, with $m d^{2}$ parameter different by a factor of $\sim 2.5$. Consequently in Cytochrome $b_{6} f$ fixed-temperature recovery and thermocycling experiments preferentially probe different components of the barrier- and $\lambda$-distributions encoded into the spectral holes. Both components most likely represent dynamics of the protein and not of the surrounding buffer/glycerol glass.
\end{abstract}




\section{Introduction.}

Spectral hole burning has been used for many years to explore energy landscapes and lowtemperature dynamics in glasses, polymers and proteins ${ }^{1-3}$. Information on these landscapes can be obtained from observing the hole burning process, the recovery of the spectral holes and their broadening at fixed (burn) temperature as well as recovery and broadening upon temporarily elevating the temperature (thermocycling) ${ }^{2,4-7}$. Consistent analysis of the data obtained for the same pigment / host system in all these experiments allows for determining the shapes and the parameters of the distributions of barriers on the energy landscape and of the tunneling parameter $\lambda$, as well as for making inferences about the nature of the entities whose structural rearrangement is responsible for non-photochemical spectral hole burning (NPHB) or for spectral shifts observed in single molecule or single protein complex experiments ${ }^{8,9}$. In the case of NPHB, the pigment molecule is not chemically photo-transformed; it is the pigment's environment that experiences some conformational change triggered by the optical excitation of a pigment ${ }^{3}$. This change can be represented by crossing a barrier on a protein energy landscape, for simplicity represented by a double-well potential (TLS, two-level system, insert in Figure 1A). The barriers are much lower when the pigment is in the excited electronic state, so they could be crossed with reasonable probability $\left(\sim 10^{-4}\right)$ within the excited state lifetime of several nanoseconds. The parameters of the ground state barriers determine how long it takes the spectrum to return to the pre-burn state (multiple hours to days at cryogenic temperatures). At low enough temperatures, barriers are crossed predominantly by tunneling, with the (downhill) transmission rate expressed as ${ }^{10-15}$,

$$
k_{0}=\Omega_{0} \exp (-2 \lambda)(n(\Delta, T)+1)
$$


where $\Omega_{0}$ is the constant attempt frequency and $n$ is the population number of phonons satisfying the energy balance condition, $T$ is temperature and $\Delta$ is asymmetry of the TLS. The tunneling parameter $\lambda$ represents a composite "barrier strength for the purpose of tunneling" and is usually reported in papers on NPHB ${ }^{3,10-15}$ as

$$
\lambda=d \sqrt{2 m V} / \eta
$$

where $V$ is the height of the energy barrier above the energy of the tunneling particle, $d$ is the distance between the two energy minima along the generalized coordinate, and $m$ is the effective mass of the entity that rearranges during the transitions between two conformational states of the protein (or other amorphous solid).

Pigment-protein complexes involved in photosynthesis have been the focus of intensive research for decades as they are important from the renewable energy perspective and exhibit a broad variety of exciting phenomena, including protein dynamics, excitation energy transfer, charge transfer, excitonic effects and even quantum coherencies. Cytochrome $b_{6} f\left(C y t b_{6} f\right)$, with its one chlorophyll $a$ per protein monomer ${ }^{16}$, is a very simple chlorophyll-protein complex, and exploring NPHB in $\mathrm{Cyt}_{6} \mathrm{~b}_{6}$ offers insights that may be useful for analyzing NPHB and single complex spectroscopy data for other, multi-pigment, complexes. In the case of Cyt $b_{6} f$ earlier NPHB data suggested a "glass-like" $\sim 1 / \sqrt{V}$ barrier distribution ${ }^{11}$. In ${ }^{11}$ we noticed that while the shapes of the distributions derived from the fixed-temperature recovery and thermocycling experiments on Cyt $b_{6} \mathrm{f}$ (and in CP43, where all results suggested Gaussian distribution ${ }^{10}$ ) were qualitatively consistent, their widths were not compatible. To resolve this problem, we proposed a radical reduction of both the attempt frequency and tunneling parameter $\lambda$, attributable to the cooperative effects ${ }^{11}$. However, we were unable to find a combination of parameters that would result in a satisfactory agreement with the experiment. Here we report additional observations, 
alternative explanation for our earlier results and the first simulations of NPHB and subsequent hole recovery involving two competing channels.

\section{Experimental Methods.}

The Cyt $b_{6}$ f samples were from the same batch as in ${ }^{11}$; n-Octyl $\beta$-D-glucopyranoside (OGP) detergent was used to solubilize the protein. Due to lower electron-phonon coupling OGP-solubilized Cyt $b_{6} f$ offers wider dynamic range in terms of possible fractional hole depths than n-Dodecyl $\beta$-D-maltopynanoside (DM)-solubilized one ${ }^{17}$. Prior to the experiment the protein solution was mixed with glycerol acting as a cryoprotectant and facilitating formation of a transparent glassy solid at low temperatures. The samples were placed in a plastic cuvette with $4 \times 10 \mathrm{~mm}$ cross-section inside a liquid helium cryostat. High-resolution spectra were measured in fluorescence excitation mode using Sirah Matisse-DS frequency-stabilized tunable dye laser (bandwidth $\sim 0.15 \mathrm{MHz}$ ). Fluorescence was detected with a Hamamatsu photomultiplier / photon counting module equipped with a long-pass filter (AELP700, Omega Filters, VT). Thermocycling experiments involve producing a spectral hole at low (burn) temperature, e.g. 5 $\mathrm{K}$, temporary increasing the temperature and allowing some systems to cross their respective barriers and return to their pre-burn state, measuring the hole spectrum again at low (burn) temperature, increasing the temperature to a slightly higher values than in the previous cycle, and so on. Simulations of competitive NPHB and recovery were performed on a personal computer with 32 GB of RAM.

\section{Results.}

Figure 1 presents an example of the hole growth kinetics (HGK) data (Frame A, closely resembling data from ${ }^{11}$ ) as well as the data on fixed-temperature $(5 \mathrm{~K})$ recovery of spectral holes 
of different initial depths (Frame B). The data in the insert suggests the presence of nearly perfect spectral memory ${ }^{11,12}$ - the pigment-protein systems recover by returning to the pre-burn conformation. The recovery data is inconsistent with the Gaussian shape of the $\lambda-$ or $V$ -

distribution (dashed lines). Recovery of a 30\%-deep hole at $5 \mathrm{~K}$ was once followed for 30 hours (main frame B; open symbols). Solid blue symbols and solid blue line in the main frame are the same as in the insert.
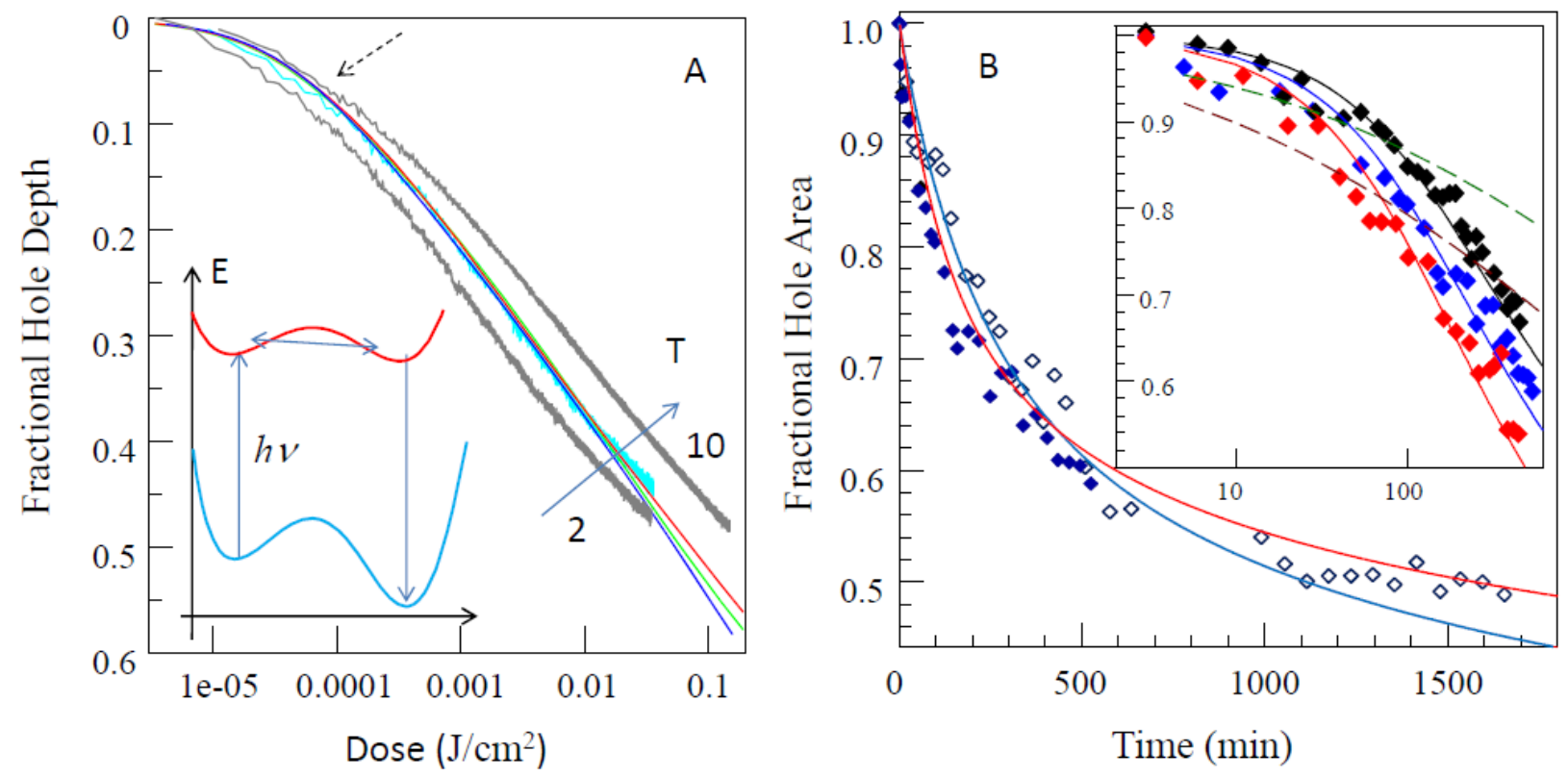

Figure 1. Frame A: HGK curves for 2, 5, and $10 \mathrm{~K}$ from ${ }^{11}$. Dashed arrow indicates the region in the beginning of the burn where at $10 \mathrm{~K}$ and above the burning is faster than tunneling-based model predicts, likely due to the onset of burning via barrier-hopping ${ }^{11}$. Also presented are the fits to the $5 \mathrm{~K}$ data (light blue). Blue: single-tier fit from ${ }^{11}$. Red: two components, both $\sim 1 / \sqrt{V}$. Green: two components, dominant $\sim 1 / \sqrt{V}$, another is Gaussian. Insert depicts the TLS model representing a pigment-protein system. Frame B: Recovery of two 30\%-deep spectral holes (open and closed symbols) and the fits assuming presence of spectral memory and $\sim 1 / \sqrt{V}$ barrier distribution. Blue curve is the fit resulting from single-tier model, red curve is obtained using two-tier model, see text. Insert: Fixed-temperature recovery data from ${ }^{11}$ for $20 \%$ (red), 30\% (blue) and 40\%-deep (black) holes with fits employing $\sim 1 / \sqrt{V}$ model (solid curves) and Gaussian $\lambda$-distribution model (dashed lines) with parameters following from the fits to HGK data. 
Figure 2A shows the results of two thermocycling experiments, both starting with a hole of $30 \%$ fractional depth, far from the saturated depth of about 50\%. In the first experiment (black squares) the hole was allowed to recover at fixed temperature $(\sim 5 \mathrm{~K})$ for an hour before thermocycling was initiated. A thermally-induced recovery step with the magnitude of about 10$15 \%$ of the original hole area was present in the temperature range around $11-15 \mathrm{~K}$ (left vertical arrow). In the second experiment (blue circles) the hole was allowed to recover at $5 \mathrm{~K}$ for ten hours before thermocycling was initiated. From the second to the tenth hours at $5 \mathrm{~K}$ the hole experienced significant recovery, comparable in magnitude to the step described above. However, subsequent thermocycling still resulted in a recovery step at around 11-15 K (right vertical arrow), with approximately the same magnitude. This indicates that ten hours long recovery at $5 \mathrm{~K}$ did not include the respective systems, but these systems were easily accessible with a small temperature increase.
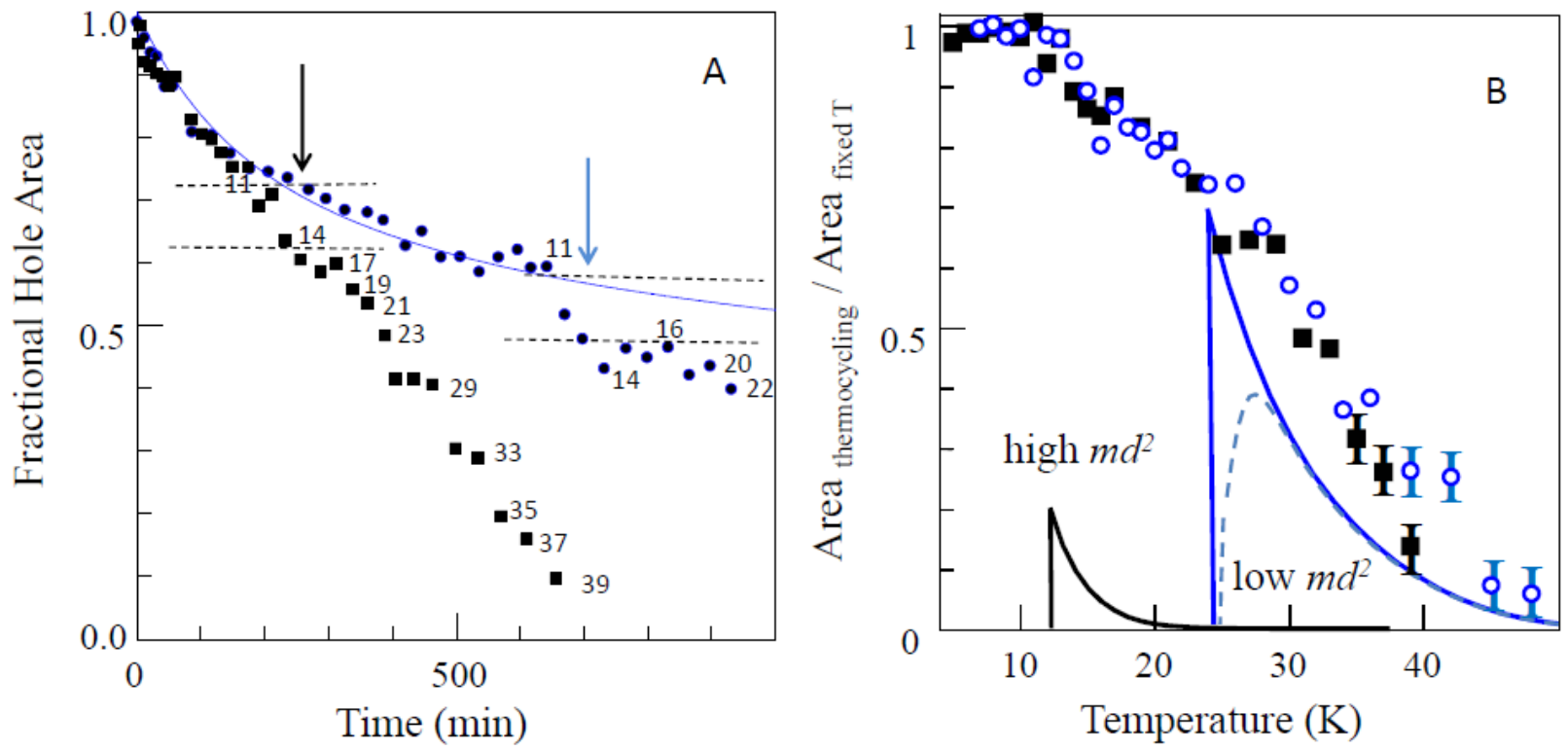

Figure 2: Frame A: Recovery of a 30\% hole with thermocycling started one hour (black squares) and ten hours (blue circles) after the end of burning. Solid blue curve is the f[rzi]it to 
fixed-temperature recovery data. Vertical arrows indicate the fast recovery step at 11-15 K in two datasets. Horizontal dashed lines assist in estimating the magnitude of the step. Frame B: The difference between thermocycling results (black squares in Frame A) and a fit to the fixedtemperature recovery represented as a function of temperature (black squares) and a similar dataset from an the experiment with deuterated sample ${ }^{17}$ (blue circles), illustrating repeatability and independence on deuteration of the solvent. Solid black and blue curves represent the barrier distributions encoded into the holes at the end of the burn / beginning of the recovery. Dashed blue curve indicates what is left of the "blue" distribution after the first three hours below $11 \mathrm{~K}$.

This step cannot be attributed to some experimental artifact related, for example, to the unusual performance of the cryostat at this particular temperature, as it was repeatable in Cyt $b_{6} f$ and not observed in $\mathrm{CP} 43^{10}$ or LH2 while using the same experimental setup. Thus, there most likely are two distinct contributions to the hole recovery and burning, characterized by significantly different $V$ and $m d^{2}$ values, as opposed to a single- $m d^{2}$ barrier distribution with both attempt frequency and mean of the $\lambda$-distribution being drastically reduced, as was proposed for $\mathrm{Cyt}_{6} \mathrm{f}$ earlier ${ }^{11}$. Figure $2 \mathrm{~B}$ depicts, as a function of temperature, the fraction of the evolution of spectral holes that cannot be accounted for by the hole recovery of the same duration at $5 \mathrm{~K}$. It is obtained by dividing the thermocycling data in Frame A (black squares) by the fit to fixed-temperature recovery (blue curve). It is a common practice to fit the data such as presented in Figure 2B to the (derivative of the) barrier distribution(s) ${ }^{4-6}$. However, more rigorous procedure involves modeling data such as shown in Figure 2A while using Eq. 3 and the temperature change profile $T(t)$ of the actual experiment.

Figure 3 summarizes the two-component model qualitatively explaining the data in Figures 1 and 2 as well as in Refs 11 and 17. NPHB is simultaneously (and possibly competitively, see below) probing two distinct barrier distributions with two different generalized coordinates and with overlapping ranges of NPHB yields (combinations of excited- 
state $\lambda$ and $\Omega_{0}$ ) - one with lower barriers $V$ and higher $m d^{2}$ (black) and the other with lower $m d^{2}$ and higher $V$ (blue). The lower $m d^{2}$ higher $V$ tier (blue) may feature either systematically higher values of NPHB yield (i.e. lower $\lambda$, as shown in Figure 3) or just broader NPHB yield distribution ensuring that the lower- $\lambda$ end of the combined distribution features lower $m d^{2}$ and so there is no significant burning by barrier-hopping in the excited state at $5 \mathrm{~K}$. In thermocycling experiments the lower-barrier higher- $m d^{2}$ part (black) is the first to recover (note the switching of the black and the blue curves between parts A, C, E and B, D, F of Figure 3).
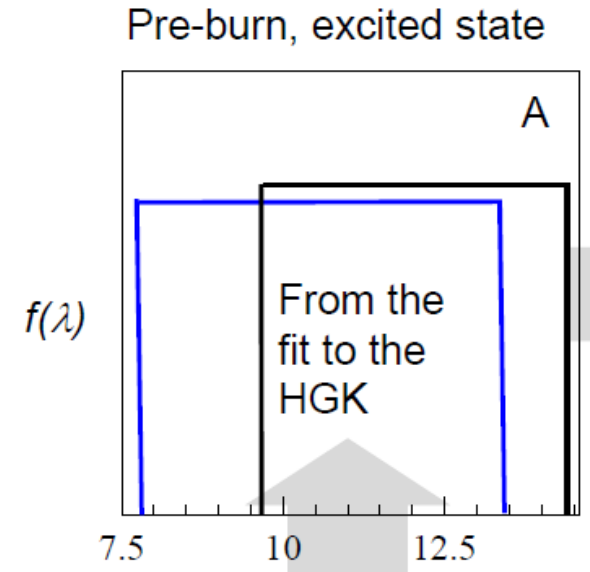

12.5

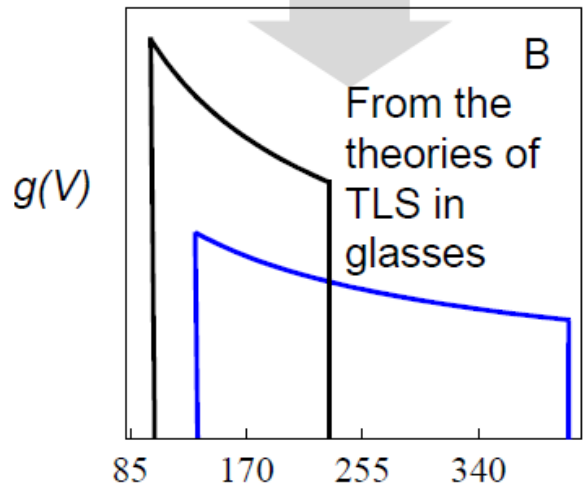

Post-burn, excited state

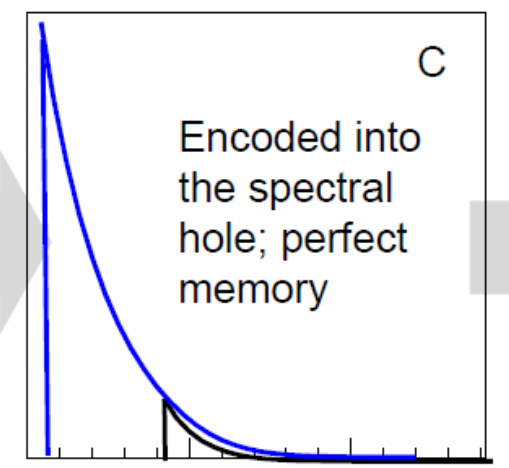

7.5

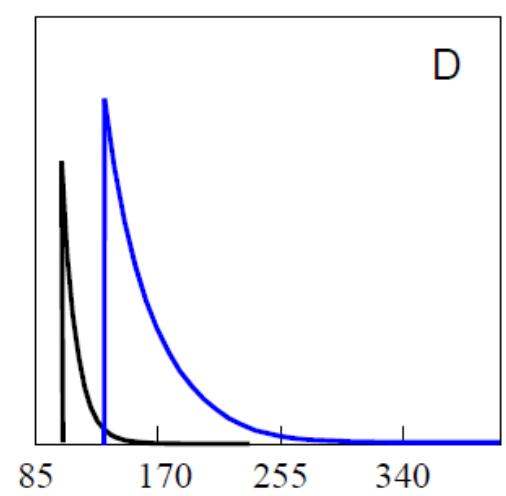

Post-burn, ground state
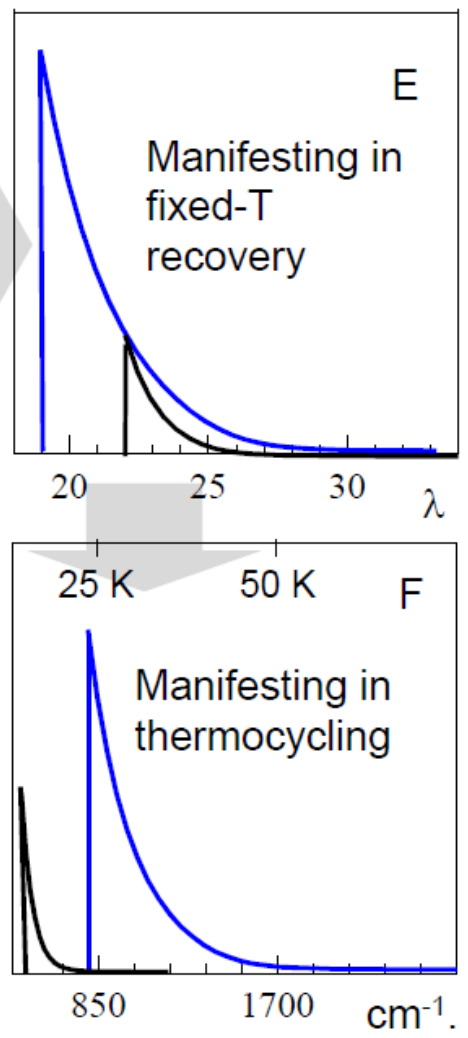

Figure 3. Likely relationship between two distributions (and sub-distributions encoded into the holes in the case of a perfect spectral memory) affecting NPHB and hole recovery in Cyt $b_{6}$ f. Frames A and B depict full true $\lambda$-distributions and barrier distributions, respectively. Frames $\mathrm{C}$ and $\mathrm{D}$ represent partial distributions encoded into the holes. Frames $\mathrm{E}$ and $\mathrm{F}$ represent the ground-state distributions manifesting in recovery experiments. The true full pre-burn $\lambda$ - 
distributions for the ground state (not shown) would look like A and B but with the horizontal scale from E and F, respectively. Blue: lower $m d^{2}$ higher- $V$ component. Black: higher $m d^{2}$ lower- $V$ component. Note the switch between "blue" and "black" components between upper and lower rows of the figure. $\lambda_{g r} / \lambda_{\text {exc }}$ is slightly different for two tiers, see Frames $\mathrm{C}$ and $\mathrm{E}$. $\lambda_{g r} / \lambda_{\text {exc }, \text { blue }}=2.46$ and $\lambda_{g r} / \lambda_{\text {exc }, \text { black }}=2.30$. See text for more details.

During the recovery the spectral holes evolve in such manner that partial $\lambda$-distributions encoded into the holes (such as those in Figure 3E) are multiplied by

$$
q\left(\lambda_{g r}, V_{g r}, t, T\right)=\exp \left(-\Omega_{0} t\left[\left(2 n_{g r}\left(\Delta_{g r}\right)+1\right) \exp \left(-2 \lambda_{g r}\right)+2 \exp \left(-\frac{V_{g r}}{k_{B} T}\right)\right]\right)
$$

This function is adopted from ${ }^{18}$ and modified to include both tunneling (the first term in the square brackets) and barrier-hopping (the second term) ${ }^{10,11} ; n_{g r}\left(\Delta_{g r}\right)$ is the average population number for relevant phonons, determined by the asymmetry of the ground-state TLS. Attentive reader may notice that Eq. 3 contains extra factors of 2 in front of the $n_{g r}\left(\Delta_{g r}\right)$ and the hopping probability, compared to similar equation from ${ }^{10,11,17}$. This modification reflects the fact that recovery of the spectral hole involves evolution of the system towards equilibrium, which must occur with the rate that is the sum of the "uphill" and "downhill" rates ${ }^{19}$. For barrier-hopping, for the sake of simplicity it was assumed that as long as the barrier height is much larger than the excited state asymmetry, the $1 \rightarrow 2$ and $2 \rightarrow 1$ rates for barrier hopping are nearly equal. Spectral hole recovers because the ensemble of the TLS evolves towards equilibrium. There is nothing fundamentally special about spectral holes, they are just a particular case of an ensemble of the pigment/environment TLS being out of equilibrium ${ }^{20}$. A sub-ensemble of the pigment-protein systems with similar electronic transition energies does not "know" if it belongs to some spectral hole or not while its populations are evolving towards equilibrium. Eq. 3 describes the probability that burnt systems with given $\lambda_{g r}$ and $V_{g r}$ will remain unrecovered after time $t$ at 
temperature $T$. The $q\left(\lambda_{g r}\right)$ is a relatively sharp (but not infinitely sharp, as in earlier, simpler models) step-like function with zero at low $\lambda_{g r}$ and one at high $\lambda_{g r}$. This function is shifting towards higher $\lambda_{g r}$ and $V_{g r}$, cutting more off the post-burn $\lambda$-distribution, with the increase in time $t$ and it shifts more within the same time with the increase of $T$.

The data from the thermocycling experiment following ten hours of fixed-temperature recovery (Figure 2A) suggests that one minute at $12 \mathrm{~K}$ had larger effect on the sub-distribution of barriers responsible for this particular recovery step (black distribution in Figure 2B and 3) than 600 minutes of recovery at $5 \mathrm{~K}$ and therefore, approximately,

$$
600 \min \cdot \exp \left(-2 \lambda_{g r}\right)\left(2 n_{g r}(12 K)+1\right) \leq 1 \min \cdot 2 \exp \left(-\frac{V_{g r}}{k_{B} 12 K}\right)
$$

Here we made an assumption that the ground-state attempt frequency is the same for tunneling and barrier-hopping. Equality corresponds to a situation when after ten hours of recovery at $5 \mathrm{~K}$ the $q$-curve (Eq. 3), moving towards the larger $\lambda_{g r}$, has reached exactly the $\lambda_{g r \text {,min }}$ of this subdistribution. In the case of the thermocycling, $V\left(T_{\max }\right)=k_{B} T_{\max } \ln \left(t_{\max } \Omega_{0}\right)^{4-6}$, where $t_{\max } \approx 60 \mathrm{sec}$ is the time spent at the peak cycle temperature $T_{\max }$. Due to the logarithm, the conversion between the temperature at which a subset of systems recovers and the respective barrier heights has weak dependence on the attempt frequency. Thus, for a broad range of reasonable $\Omega_{0}$ around $10^{11}-10^{13}$ $\mathrm{Hz}$, the systems recovering at $\sim 12 \mathrm{~K}$ (black distributions in Figure 3) feature barriers of $\sim 370 \mathrm{~cm}^{-}$ ${ }^{1}$ with at most $\pm 10 \%$ uncertainty and $\lambda_{g r, m i n} \approx 25$ for these systems. Additionally the other tier of the energy landscape (blue), responsible for the observable hole recovery at $5 \mathrm{~K}$, must feature somewhat smaller $\lambda_{g r, m i n} \approx 20$. These values of $\lambda_{g r}$ are in reasonable agreement with the data reported for hyperquenched glassy water ${ }^{15}$.

Note that the above estimate does not require any knowledge of the excited state TLS parameters that could be obtained from the fit to the HGK (Figure 1A). Given the two-tier model 
shown in Figure 3, one should consider if the NPHB is competitive (both channels are available for each system) or one or another NPHB channel exists in a given individual pigment-protein system. The latter assumption would imply that our samples contained two distinct subpopulations of pigment-protein systems with very different $m d^{2}$. It is unlikely that two distinct local environments of a Chl $a$ molecule, with different conformational changes available to them, would exist in Cyt $b_{6} f$ where all Chls are located in identical protein pockets ${ }^{16}$. In any case, NPHB in such a scenario can be easily modeled as a sum of burning into two independent components. The competitive NPHB on two different tiers of the protein energy landscape can be modeled assuming that NPHB yields for two competing hole burning processes will be $\phi_{1}\left(\lambda_{1}, \lambda_{2}\right)=\frac{\Omega_{01} \exp \left(-2 \lambda_{1}\right)}{\Omega_{01} \exp \left(-2 \lambda_{1}\right)+\Omega_{02} \exp \left(-2 \lambda_{2}\right)+\tau_{1}^{-1}} ; \quad \phi_{2}\left(\lambda_{1}, \lambda_{2}\right)=\frac{\Omega_{02} \exp \left(-2 \lambda_{2}\right)}{\Omega_{02} \exp \left(-2 \lambda_{1}\right)+\Omega_{02} \exp \left(-2 \lambda_{2}\right)+\tau_{1}^{-1}}$ where $\Omega_{0}$ are the attempt frequencies and $\tau_{1}$ is the fluorescence lifetime. The total NPHB yield will be $\phi\left(\lambda_{1}, \lambda_{2}\right)=\phi_{1}+\phi_{2}$. Contributions of the two tiers of the energy landscape to any spectral hole would scale as $\phi_{1}$ to $\phi_{2}$.

The HGK curves behave as in the case of a single-component $\lambda$-distribution with significantly smaller width. For instance, a reasonable fit to 5 K HGK curve in Figure 1 can be produced with a combination of two box-like $\lambda$-distributions, one ranging from 7.8 to 13.2 and another from 9.5 to 14.7 . For the sake of simplicity and comparability, the same value of the attempt frequency as in ${ }^{10,11,17}, 7.6 \cdot 10^{12} \mathrm{~s}^{-1}$ was used for both tiers. The first (blue) $\lambda$-distribution is significantly broader than a single $\lambda$-distribution that was required for fitting the same HGK data ( 7.8 to $12.0^{11}$, i.e. 4.2 units versus 5.4 above). Note that in the non-competitive burning scenario, involving two distinct populations with different conformational changes, the distributions will be narrower. Two partial distributions produced with the above parameters and 
encoded into the 30\% holes are actually those shown in Figure 3E and F. If there is spectral memory, these partial distributions are probed in the recovery experiments, not the full ones in Figure $3 \mathrm{~A}, \mathrm{~B}$. The area under the black curve is about $10 \%$ of the total area under both curves combined, in agreement with the magnitude of the recovery step observed at around 11-15 K. The two-component model offers slightly better fit to the fixed-temperature recovery data, especially at long times, and yields $\lambda_{g r} / \lambda_{e x c}=2.46$. We are not aware of a physical reason why different tiers of the energy landscape should feature exactly identical $\lambda_{g r} / \lambda_{e x c}$ ratios; so far this ratio was used as a free parameter to arrive at the best fit to the fixed-temperature recovery data $10,11,17$. Therefore, we allow for somewhat different $\lambda_{g r} / \lambda_{\text {exc }}$ for "blue" and "black" tiers of the energy landscape. Smaller $\lambda_{g r} / \lambda_{\text {exc }}$ for the "black" tier allows for somewhat smaller value of $m d^{2}$ and better fit to the HGK data at temperatures higher than $5 \mathrm{~K}$. However, $\lambda_{g r} / \lambda_{e x c}$ should not be significantly smaller for the "black" tier than for the "blue" tier: If there were more overlap between the two ground-state $\lambda$-distributions (imagine shifting black curve in Figure 3E further to the left), the "black" tier of the landscape would contribute significantly to the tunnelingdominated fixed-temperature recovery at earlier times, and after the first ten hours at $5 \mathrm{~K}$ significant portion of this component would be recovered. The minimal value of $\lambda_{g r} / \lambda_{\text {exc black }}$, i.e. the value where the fixed-temperature recovery does not yet become faster for fixed $\lambda_{g r} / \lambda_{e x c}$, blue $=2.46$, is 2.30 . Figure 4 demonstrates the fits to fixed-temperature recovery and thermocycling data, obtained utilizing the temperature change profile of the real experiment and yielding $m d^{2}$ blue $=1.5 \cdot 10^{-46} \mathrm{~kg} \mathrm{~m}^{2}$ and $m d^{2}$ black $=4.0 \cdot 10^{-46} \mathrm{~kg} \mathrm{~m}^{2}$. The fit to the thermocyling data captures most of the details; possible reasons for this discrepancy are considered in the Discussion section. 


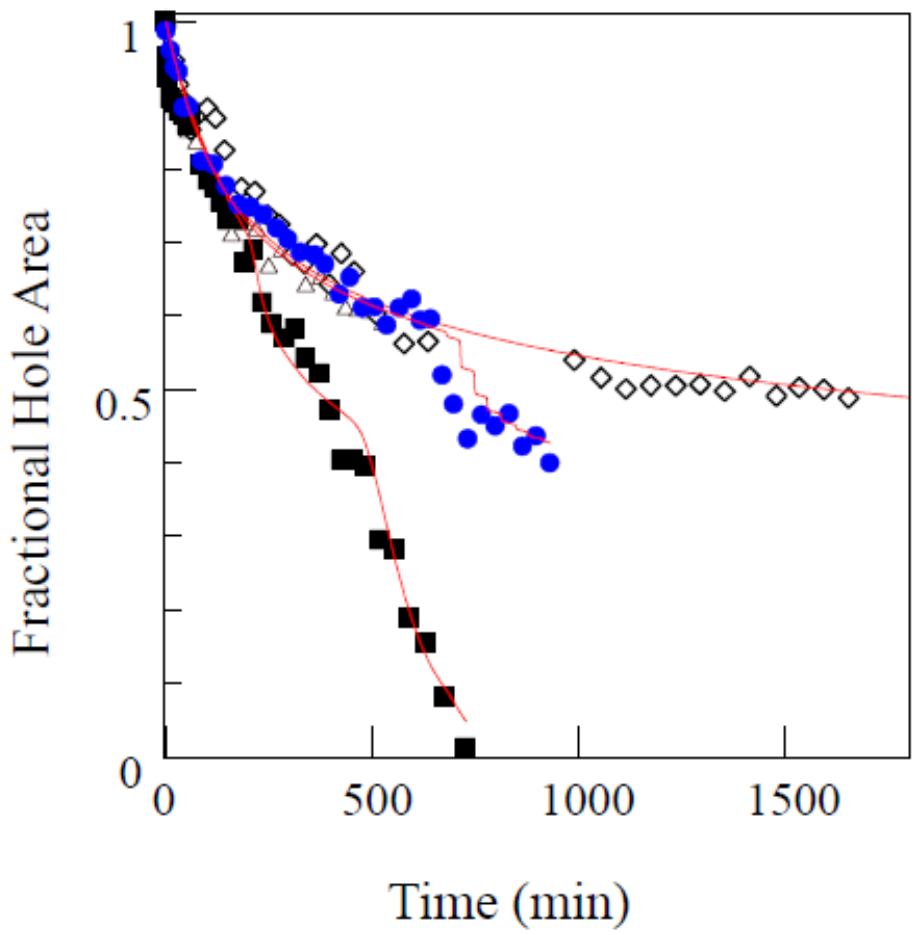

Figure 4. Fixed-temperature recovery data (open symbols) from Fig. 1B and thermocycling data (filled symbols) from Fig. $2 \mathrm{~A}$ with the fits obtained with $m d^{2}$ blue $=1.5 \cdot 10^{-46}$ $\mathrm{kg} \cdot \mathrm{m}^{2}$ and $m d^{2}{ }_{\text {black }}=4.0 \cdot 10^{-46} \mathrm{~kg} \cdot \mathrm{m}^{2}$ using the temperature change profiles of the respective experiments (red curves).

It is impossible to achieve the satisfactory global fit to the available data when the preburn $\lambda$-distributions are Gaussian since it is more difficult to achieve the required separation of the ground-state barrier distributions encoded into the holes. According to Figure $1 \mathrm{~B}$ and ${ }^{11}$ the dominant contribution to recovery (blue) is not a descendant of a Gaussian anyway. However, given the uncertainties of the thermocycling data, one could argue that the second distribution (black) could still be Gaussian. A satisfactory fit to the 5 K HGK data in Figure 1 (good fit for the first $40 \%$, smooth green curve) can be obtained starting from a box-like $\lambda$-distribution in the 7.8-13.0 range (blue) plus a Gaussian $\lambda$-distribution peaked at 11.7 and with STD $\sigma_{\lambda}=1.0$ (black). For 30\%-deep hole this results in the "black" tier contributing about $13 \%$ to the hole. 
Note that the lower- $\lambda$ tail of the Gaussian (black) component reaches further than the $\lambda_{\min }$ of the "blue" component. In order to achieve sufficient separation between the ground-state distributions encoded into the holes, and to allow for no or almost no recovery of the "black" component in the first ten hours at $5 \mathrm{~K}$, the $\lambda_{g r} / \lambda_{\text {exc }}$ value for the "black" tier must not be smaller than for the "blue" tear, unlike in the case when one starts from two box-like $\lambda$-distributions. This requires a higher value of $m d^{2}$ of at least $4.5 \cdot 10^{-46} \mathrm{~kg} \cdot \mathrm{m}^{2}$ to compensate and to ensure that the "black" tier still experiences recovery upon thermocycling at a correct temperature, which in turn leads to the earlier onset of spectral hole burning via barrier hopping, in disagreement with experiment.

\section{Discussion.}

As demonstrated above, introducing simultaneous NPHB involving two tiers of the protein energy landscape that are partially overlapping in terms of $\lambda$ but are separate in terms of the postburn barrier distributions offered a natural solution to the problem of the mismatch between the

widths of the $\lambda$ - and barrier distributions deduced from the experimental results ${ }^{10,11}$. Competitive burning proved to be helpful as well, as it allowed for starting from the broader $\lambda$-distributions when modeling the HGK data. However, arriving at a single set of parameters that would explain all observations quantitatively still proved very difficult. Thus, below we consider possible avenues of further improving the procedures for modeling the NPHB and recovery data.

\subsection{The Details of the NPHB Process and Implications for Spectral Memory.}

In the previous section we suggested that the recovery of the spectral holes should be interpreted as evolution of the ensemble of pigment-protein systems towards thermodynamic 
equilibrium. This resulted in additional factors of two in Eq. 3, taking into account that evolution towards equilibrium occurs with the rate that is the sum of the "uphill" and "downhill" rates ${ }^{19}$. Would similar argument be valid for the hole burning process, involving the excited state? First, we stress that the following discussion is applicable to each of the "blue" and "black" components in Figure 3, one should not interpret it as if we were are considering one of these components as memory-less and the other exhibiting perfect spectral memory. One can split the ensemble of the individual pigment-protein systems resonant with the laser into two subensembles, one where upon excitation the individual systems end up in the lower well of the excited-state TLS and the other where they find themselves in the higher well of the excited-state TLS. Each of these two sub-ensembles will be out of thermodynamic equilibrium, and will evolve with the rate, which is the sum of the "uphill" and "downhill" rates for the excited state ${ }^{19}$. Can this evolution towards equilibrium argument still be valid given the short (nanoseconds) lifetime of the excited state? One could note that tunneling model used in this and earlier NPHB works started from free-particle wavefunctions. In reality, the wavefunctions in each well of the TLS must be closer to the wavefunctions of the harmonic oscillator ${ }^{21,22}$ and the well populations would oscillate back and forth. Additionally, as demonstrated in ${ }^{20}$, realistic cooling may result in the ensemble being not in thermodynamic equilibrium prior to burning. And even in equilibrium, some systems will be found in the upper well of the ground-state TLS before burning. Thus, the recovery of any spectral hole may exhibit some small memory-less contribution and the respective distributions manifesting in hole recovery may have both components. The magnitude of the memory-less component will depend on the pre-burn population of the higher well of the ground state TLS, i.e. on the ground state asymmetry, as well as on the history of the experiment / cooling procedure ${ }^{20}$. Since the component featuring spectral memory must be dominated by 
smaller barriers or $\lambda^{10,11}$ (Figure 3C), initial phases of recovery will still be dominated by the component with memory. Small memory-less contribution may provide somewhat more gradual recovery upon thermocycling, in better agreement with the experimental results.

According to Figure 1A, in the excited state tunneling dominates in the NPHB process until about $10 \mathrm{~K}$. Thus,

$$
\exp \left(-2 \lambda_{e x, \min }\right)\left(2 n_{e x}(10 K)+1\right)>2 \exp \left(-\frac{V_{e x, \min }}{k_{B} 10 K}\right)
$$

and $-2 \lambda_{e x, \min }+\ln \left(2 n_{e x}(10 K)+1\right)>-\frac{V_{e x, \min }}{7}+\ln 2$. The weak temperature dependence of the HGK (Figure 1A) indicates that the excited state TLS asymmetries are not lower than $4 \mathrm{~cm}^{-1}$ $\left(4.5 \mathrm{~cm}^{-1}\right.$ were used in our simulations). Excited state asymmetry as high as $27 \mathrm{~cm}^{-1}$ was proposed for hyperquenched glassy water ${ }^{15}$. For large enough asymmetries both logarithm terms are smaller than other terms and partially cancel each other. Thus, $V_{\text {ex,min }} / \lambda_{\text {ex,min }} \gtrsim 14$ ?, which for $\lambda_{e x, \min } \sim 7.8$ and 9.5 yields $V_{e x, \min } \geq 110$ (blue) and $130 \mathrm{~cm}^{-1}$ (black), respectively. For the lower$V_{g r}$ tier of the energy landscape (black) $\lambda_{g r} / \lambda_{e x} \sim 2.3$ and $V_{g r} / V_{e x} \leq 370 / 130 \sim 2.8$. These ratios of $\lambda$ and $V$ are clearly inconsistent with the quadratic dependence of $V$ on $\lambda$ (Eq. 2), indicating that more advanced models describing tunneling may have to be applied in this case. For the other tier (blue) $V_{g r} / V_{e x} \approx 6$ as this component exhibits recovery due to thermocycling starting from about $20 \mathrm{~K}$. In other words, for this tier $V \sim \lambda^{2}$ is possible.

Eq. 2 was derived assuming rectangular barriers and free-particle wavefunctions outside of the barrier region. For more realistic inverted parabolic barriers and while $2 \lambda>>1$, but still in the approximation where the "outer walls" of the TLS are absent and reintroduced artificially via the attempt frequency,

$$
\lambda=\frac{\pi}{4} d \sqrt{2 m V} / \eta
$$


Another model, employing parabolic wells, was recently considered in ${ }^{21}$. The wavefunctions for the ground state of the harmonic oscillator were employed, the probabilities to find the system in each of the wells oscillated out of phase, and the probability of transition per unit time was

$$
k_{0} \approx \frac{2 v}{\sqrt{\pi}} \delta \times \exp \left(-\delta^{2}\right)
$$

Note that Eq. 7 does not contain temperature dependence; we will return to this issue shortly. $\delta=d^{\prime} / 2 x_{0}$ is dimensionless, $d^{\prime}$ is the full distance between the TLS minima, $x_{0}=\sqrt{\frac{\hbar}{m \omega}}$ is the displacement of the harmonic oscillator at the lowest vibrational level, $v$ is the attempt frequency in $\mathrm{Hz}$ and $\omega$ is the same in $\mathrm{rad} / \mathrm{s}$. Comparing this with Eq. 1 suggests $\delta^{2}=2 \lambda$. Overall, in this model the quadratic relationship between $V$ and $\lambda$ is preserved, but the pre-factor in the tunneling rate expression becomes dependent on $\lambda$ as $\lambda^{3 / 2}$ (or $V^{3 / 4}$ ). Specifically, Eq. 7 can be manipulated to

$$
k_{0}=\frac{4 \lambda \hbar}{\pi m d^{2}} \frac{2 \sqrt{2 \lambda}}{\sqrt{\pi}} \exp (-2 \lambda)=\frac{1.27 * \lambda * 10^{12} \mathrm{~Hz}}{m d^{2}} 1.6 \sqrt{\lambda} \exp (-2 \lambda),
$$

where $m d^{2}$ is in the units of $10^{-46} \mathrm{~kg} \mathrm{~m}^{2}$. For $\lambda \sim 10-20$, the pre-factor falls into the range of attempt frequencies reported elsewhere when $m d^{2}$ is several times $10^{-46} \mathrm{~kg} \mathrm{~m}^{2}$. One could generalize that $\lambda=C d \sqrt{2 m V} / \hbar$ where $C=1.0$ for rectangular barriers, $C=\pi / 4$ for inverted parabolic barriers, $C \approx 1 / 4$ for parabolic wells, etc. Concerning the temperature dependence associated with Eqs. 7 and 8, the NPHB rate is weakly dependent on temperature, and the observed slowdown of the HGK (Fig. 1A) is due to the increase of the homogeneous line width $^{10,11}$. Thus, one should utilize the weak temperature dependence corresponding to phonon scattering ${ }^{10-15,17}$ (see Eq. 1) rather than the exponential dependence suggested in ${ }^{21}$.

From Eq. 4 with $V \sim \lambda^{2}$ tunneling is the dominant NPHB mechanism when 


$$
\frac{\lambda_{e x}^{2} \hbar^{2} c^{-2}}{2 m d^{2} k_{B} T}-2 \lambda_{e x}+\ln \left(n_{e x}+1 / 2\right)>0
$$

The last term is much smaller than the first two for $T=5-10 \mathrm{~K}, \lambda_{e x} \sim 10$ and reasonable excited state TLS asymmetries. As long as $C^{-2}>1$, i.e. for any barriers but the rectangular ones, larger $m d^{2}$ could be accommodated for the same $\lambda_{e x}$ without introducing barrier hopping. Unfortunately, this does not help ensure that the T-induced recovery occurs at the correct temperature, as the expression for this temperature contains the ratio of the correction factor $C^{-2}$ and $m d^{2}$.

\subsection{Distributions.}

In ${ }^{11}$ we reported a mismatch between the widths of the $\lambda$-distributions responsible for fixed-temperature recovery and thermocycling. Similar problem was in fact present for CP43 complex ${ }^{10}$. Allowing for an additional distribution component (black) in Cyt $b_{6}$ f reduces this problem. (In the case of $\mathrm{CP} 43^{10}$, availability of different conformational changes may also result from the lowest-energy state being delocalized over several chlorophylls.) Concerning the most probable shape of the "blue" component, predominantly encoded into the holes, the results presented in Figure 1B suggest that it should be uniform / box-like. HGK and recovery results obtained for $\mathrm{Cyt}_{\mathrm{b}} \mathrm{f}$ in fully deuterated buffer-glycerol environment indicate that there are very little, if any, differences in the hole burning and recovery dynamics ${ }^{17}$. We conclude that spectral dynamics on the energy landscape tier characterized by the "blue" curve in Figure 3 definitely belongs to the protein, while the dynamics on the tier characterized by the black curve most likely belongs to the protein as well, but may with some small probability also be due to the movements of the $-\mathrm{OH}$ groups in the water/glycerol solvent surrounding the protein ${ }^{17}$. The latter is fairly unlikely because the respective deuteration-induced increase of $\lambda$ by $3 \%{ }^{17}$, i.e. to $\lambda_{\min }$, $e_{x c}=9.8$ would significantly reduce the contribution of the "black" tier to the holes and render the 
step at 11-15 K unobservable. However, according to Figure 2B the step at 11-15 K was still observed in thermocycling data of the deuterated sample (blue circles). The values of $m d^{2}$ deduced from the data effectively rule out tunneling of a single proton in a double-well potential associated with a hydrogen bond as a possible source of the observed dynamics. The $m d^{2}$ in this case would be $0.7 \cdot 10^{-46} \mathrm{~kg} \mathrm{~m}^{2}$ or less, taking into account that separation of the potential wells in this scenario must be smaller than the typical hydrogen bond length. Cooperative effects involving the whole hydrogen bonds network of the protein are still possible, since in this case $m$ no longer has to be equal to the mass of a single proton. Alternatively, this component could represent a rearrangement of some relatively light chemical group such as $-\mathrm{CH}_{3},-\mathrm{OH}$, etc. Note that a mechanism involving rotations of the methyl group by 120 degrees is unlikely, as those have a characteristic signature in the NPHB data ${ }^{23,24}$ that we did not observe.

\section{Conclusions.}

In cytochrome $b_{6} f$ the relevant distribution of the tunneling parameter $\lambda$ is a superposition of two contributions, which are significantly overlapping in terms of the resultant NPHB yield and represent two tiers of the energy landscape responsible for NPHB. However, similar values of the NPHB yield result from distinctly different combinations of barrier heights, the shifts along the generalized coordinate and/or the masses of the entities involved in conformational changes. Specifically, $m d^{2}$ of $1.5 \pm 0.1 \cdot 10^{-46} \mathrm{~kg} \mathrm{~m}^{2}$ (blue) and $4.0 \pm 0.3 \cdot 10^{-46} \mathrm{~kg} \mathrm{~m}^{2}$ (black) were deduced for these two tiers. Consequently, fixed-temperature recovery and thermocycling experiments preferentially probe different components of the barrier- and $\lambda$-distributions encoded into the spectral holes. The "blue" component likely represents the cooperative dynamics of the protein, while the "black" one may originate from several sources within the 
protein, as well as, though not likely, the $-\mathrm{OH}$ group dynamics of the solvent surrounding the protein. Both tiers feature barrier distributions of the $\sim 1 / \sqrt{V}$ type (uniform / box-like $\lambda$ distribution) prior to burning. One should not assume that other orthogonal generalized coordinates with significantly different $\lambda$ cannot exist on a real multi-well and multidimensional protein energy landscape. They definitely can. However landscape tiers with too small $\lambda$ may yield only transient holes and/or hole broadening, while the barriers of the landscape tiers with too large $\lambda$ are very unlikely crossed with non-negligible probability when easier-burning landscape tiers are available. There are also indications that for the "black" tier of the energy landscape $V \sim \lambda^{2}$ (Eq. 2) is not valid. Further improvements in the interpretation and modeling of the NPHB data, including the data on hole recovery and thermocycling, require adopting more rigorous treatment of tunneling in biological systems, beyond the WKB approximation.

\section{Acknowledgments.}

Work at Concordia University was supported by NSERC, CFI and Concordia seed grants. R.P. and M.A.L. acknowledge support by the Ministry of Economy and Competiveness of Spain (Grant No. AGL2014-55300-R) and Aragon Government (Grant E33). 


\section{References.}

1. Wagie, H. E.; Geissinger, P. Hole-Burning Spectroscopy as a Probe of Nano-environments and Processes in Biomolecules: A Review, Appl. Spectrosc. 2012, 66, 609-627.

2. Berlin, Y.; Burin, A.; Friedrich J.; Köhler, J. Spectroscopy of Proteins at Low Temperature. Part I: Experiments with Molecular Ensembles; Phys. Life Rev. 2006, 3, 262-292.

3. Jankowiak, R.; Hayes, J. M.; Small, G. J. Spectral Hole-Burning Spectroscopy in Amorphous Molecular Solids and Proteins, Chem. Rev. 1993, 93, 1471-1502.

4. Köhler, W.; Friedrich, J. Distribution of Barrier Heights in Amorphous Organic Materials, Phys. Rev. Lett. 1987, 59, 2199-2202.

5. Köhler, W.; Meiler, J.; Friedrich, J. Tunneling Dynamics of Doped Organic Low-temperature Glasses as Probed by a Photophysical Hole-burning System, Phys. Rev B 1987, 35, 40314037.

6. Köhler, W., Friedrich, W., Scheer, H., Conformational Barriers in Low-temperature Proteins and Glasses. Phys. Rev. A 1988, 37, 660-662.

7. den Hartog, F. T. H.; van Papendrecht, C.; Störkel, U.; Völker, S. Protein Dynamics in Photosystem II Complexes of Green Plants Studied by Time-Resolved Hole-Burning. J. Phys. Chem. B 1999, 103, 1375-1380.

8. Hofmann, C.; Aartsma, T. J.; Michel H.; Köhler, J. Direct Observation of Tiers in the Energy Landscape of a Chromoprotein: A Single-molecule Study; Proc. Natl. Acad. Sci. USA 2003, $100,15534-15538$.

9. Berlin, Y.; Burin, A.; Friedrich, J.; Köhler, J. Low Temperature Spectroscopy of Proteins. Part II: Experiments with Single Protein Complexes; Phys. Life Rev. 2007, 4, 64-89. 
10. Najafi, M.; Herascu, N.; Seibert, M.; Picorel, R.; Jankowiak, R.; Zazubovich, V. Spectral Hole Burning, Recovery, and Thermocycling in Chlorophyll-Protein Complexes:

Distributions of Barriers on the Protein Energy Landscape, J. Phys. Chem. B 2012, 116, $11780-11790$.

11. Najafi, M.; Herascu, N.; Shafiei, G.; Picorel, R.; Zazubovich, V. Conformational Changes in Pigment-Protein Complexes at Low Temperatures - Spectral Memory and a Possibility of Cooperative Effects, J. Phys. Chem. B 2015, 119, 6930-6940.

12. Reinot, T.; Dang, N. C.; Small, G. J. A Model for Persistent Hole Burned Spectra and Hole Growth Kinetics that Includes Photoproduct Absorption: Application to Free Base Phthalocyanine in Hyperquenched Glassy Ortho-dichlorobenzene at 5 K. J. Chem. Phys. 2003, 119, 10404-10414.

13. Reinot, T.; Small, G. J. Modeling of Dispersive Nonphotochemical Hole Growth Kinetics Data. Al-phthalocyanine Tetrasulphonate in Hyperquenched Glassy Water, J. Chem. Phys. 2000, 113, 10207-10214.

14. Reinot, T.; Zazubovich, V.; Hayes, J. M.; Small, G. J. New Insights on Persistent Nonphotochemical Hole Burning and Its Application to Photosynthetic Complexes, J. Phys. Chem. B 2001, 105, 5083-5098.

15. Dang, N. C.; Reinot, T.; Reppert, M.; Jankowiak, R. Temperature Dependence of Hole Growth Kinetics in Aluminum-Phthalocyanine-Tetrasulfonate in Hyperquenched Glassy Water, J. Phys. Chem. B 2007, 111, 1582-1589.

16. Yamashita, E., Zhang, H., Cramer, W.A., Structure of the Cytochrome $b_{6} f$ complex: Quinone Analogue Inhibitors as Ligands of Heme cn. J. Mol. Biol. 2007, 370, 39-52. 
17. Levenberg, A.; Shafiei, G.; Lujan, M.A.; Giannacopoulos,S.; Picorel, R.; Zazubovich, V. Probing Energy Landscapes of Cytochrome $b_{6} f$ with Spectral Hole Burning: Effects of Deuterated Solvent and Detergent, J. Phys. Chem. B 2017, 121, 9848-9858.

18. Love, S. P.; Mungan, C. E.; Sievers A. J.; Campbell, J. A. Persistent Infrared Spectral Hole Burning of $\mathrm{Tb}^{3+}$ in the Glasslike Mixed Crystal $\mathrm{Ba}_{1-\mathrm{X}-\mathrm{YLa}} \mathrm{Tb}_{\mathrm{y}} \mathrm{F}_{2+\mathrm{x}+\mathrm{y}}$, J. Opt. Soc. Am. B 1992, 9, 794-799.

19. Nelson, P., Biological Physics - Energy, Information, Life, W. H. Freeman, NY, 2013, pp 194-197.

20. Najafi, M.; Zazubovich, V. Monte-Carlo Modeling of Spectral Diffusion Employing Multiwell Protein Energy Landscapes: Application to Pigment-Protein Complexes Involved in Photosynthesis, J. Phys. Chem. B, 2015, 119, 7911-7921.

21. Krasilnikov, P., M.; Knox P. P.; Rubin, A. B.; Relaxation Mechanism of Molecular Systems Containing Hydrogen Bonds and Free Energy Temperature Dependence of Reaction of Charges Recombination within Rhodobacter sphaeroides RC, Photochem. Photobiol. Sci., 2009, 8, 181-195.

22. Garashchuk, S.; Gu, B.; Mazzuca, J. Calculation of the Quantum-Mechanical Tunneling in Bound Potentials, J. Theor. Chem. 2014, 240491.

23. Pinsker, M.; Friedrich, J. Hole Burning Spectroscopy and Quantum Phenomena in Methyl Groups, Mol. Cryst. Liq. Cryst. 1996, 291, 97-102.

24. Pinsker, M.; Friedrich, J. Methyl Group Rotation of p-Chlorotoluene in Cyclohexane: a Nuclear Spin Conversion Study via UV-hole Burning, Chem. Phys. Lett. 2001, 341, 545-550. 


\section{TOC Graphic:}
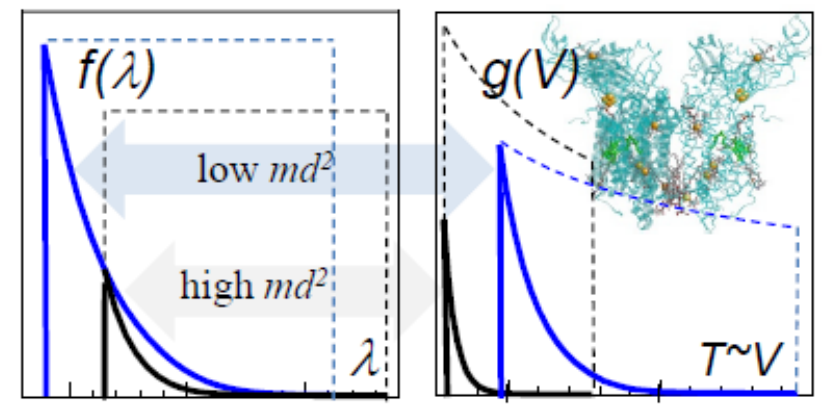